OPEN ACCESS

Edited by:

Alessandra Oliva,

Sapienza University of Rome, Italy

Reviewed by:

Renan Mauch,

State University of Campinas, Brazi

Celine Vidaillac,

University of Oxford, United Kingdom

*Correspondence:

Hamid Solgi

hamid.solgi@gmail.com

${ }^{\dagger}$ These authors have contributed equally to this work

Specialty section: This article was submitted to Clinical Microbiology, a section of the journal Frontiers in Cellular and Infection Microbiology

Received: 02 October 2021 Accepted: 28 January 2022

Published: 23 February 2022

Citation:

Pourajam S, Kalantari E, Talebzadeh H, Mellali H, Sami R, Soltaninejad F, Amra B, Sajadi M, Alenaseri $M$, Kalantari $F$ and Solgi $H$

(2022) Secondary Bacterial Infection and Clinical Characteristics in Patients With COVID-19 Admitted to

Two Intensive Care Units of an Academic Hospital in Iran During the

First Wave of the Pandemic. Front. Cell. Infect. Microbiol. 12:784130.

doi: 10.3389/fcimb.2022.784130

\section{Secondary Bacterial Infection and Clinical Characteristics in Patients With COVID-19 Admitted to Two Intensive Care Units of an Academic Hospital in Iran During the First Wave of the Pandemic}

\author{
Samaneh Pourajam ${ }^{1 \dagger}$, Elham Kalantari ${ }^{2 \dagger}$, Hamid Talebzadeh ${ }^{3}$, Hamid Mellali $^{3}$, \\ Ramin Sami ${ }^{1}$, Forogh Soltaninejad ${ }^{4}$, Babak Amra ${ }^{5}$, Mahdi Sajadi ${ }^{6}$, Malihe Alenaseri ${ }^{7}$, \\ Forough Kalantari ${ }^{8}$ and Hamid Solgi ${ }^{6,9 *}$

\begin{abstract}
${ }^{1}$ Department of Internal Medicine, School of Medicine, Isfahan University of Medical Sciences, Isfahan, Iran, ${ }^{2}$ Department of University of Medical Sciences, Isfahan, Iran, ${ }^{4}$ The Respiratory Research Center, Pulmonary Division, Department of Internal Medicine, School of Medicine, Isfahan University of Medical Sciences, Isfahan, Iran, ${ }^{5}$ Bamdad Respiratory Research Center, Isfahan University of Medical Sciences, Isfahan, Iran, ${ }^{6}$ Division of Clinical Microbiology, Department of Laboratory Medicine, Amin Hospital, Isfahan University of Medical Sciences, Isfahan, Iran, ${ }^{7}$ Infection Control Unit of Amin Hospital, Isfahan University of Medical Sciences, Isfahan, Iran, ${ }^{8}$ Department of Nuclear Medicine, Rasoul Akram Hospital, Iran University of Medical Sciences, Tehran, Iran, ${ }^{9}$ Isfahan Endocrine and Metabolism Research Center, Isfahan University of Medical Sciences, Isfahan, Iran
\end{abstract} \\ Pulmonology, Isfahan University of Medical Science, Isfahan, Iran, ${ }^{3}$ Department of Surgery, School of Medicine, Isfahan
}

Data on the prevalence of bacterial co-infections and secondary infection among adults with COVID-19 admitted to the intensive care unit (ICU) are rare. We aimed to determine the frequency of secondary bacterial infection, antibiotic use, and clinical characteristics in patients admitted to the ICU with severe SARS-CoV-2 pneumonia. This was a retrospective cohort study of adults with severe COVID-19 admitted to two ICUs from March 6 to September 7, 2020 in an academic medical center in Isfahan, Iran. To detect COVID-19, reverse transcription real-time polymerase chain reaction was performed and also typical pattern of CT scan was used for the diagnosis of COVID-19. Data collection included the age, gender, main symptoms, history of underlying disease, demographics, hospital stay, outcomes, and antibiotic regimen of the patient. Antimicrobial susceptibility testing was carried out according to the CLSI guidelines. During the study period, 553 patients were referred to the both ICUs for COVID-19 with severe pneumonia. Secondary bacterial infection was detected in 65 (11.9\%) patients. The median age was 69.4 (range 21-95) years; $42(63.6 \%)$ were men. Notably, 100\% $(n=65)$ of the patients with superinfection were prescribed empirical antibiotics before first positive culture, predominantly meropenem (86.2\%) with a median duration of 12 (range 2-32) days and levofloxacin (73.8\%) with a median duration of nine (range 2-24) days. Most prevalent causative agents for secondary bacterial infection were Klebsiella pneumoniae $(n=44)$ and Acinetobacter baumannii $(n=33)$. Most patients with secondary bacterial infection 
showed extensive drug-resistance. The mortality among patients who acquired superinfections was $83 \%$ against an overall mortality of $38.1 \%$ in total admitted COVID19 patients. We found a high prevalence of carbapenem-resistant Gram-negative bacilli in COVID-19 patients admitted to our ICUs, with a high proportion of $K$. pneumoniae followed by $A$. baumannii. These findings emphasize the importance of implementation of strict infection control measures and highlight the role of antimicrobial stewardship during a pandemic.

Keywords: bacterial co-infection, antibiotic use, COVID-19, carbapenem resistant bacteria, intensive care unit

\section{INTRODUCTION}

The severe acute respiratory syndrome coronavirus 2 (SARS-CoV2), which causes coronavirus disease 2019 (COVID-19), was first identified in December 2019 in Wuhan, China, and is currently circulating worldwide. COVID-19 disease is the greatest pandemic of our generation, with 227 million people infected and 4,664,000 deaths worldwide (WHO). The World Health Organization (WHO) guidelines for the clinical management of COVID-19 disease advise clinicians to collect specimens from the upper respiratory tract and blood samples for bacterial cultures, and to start empirical antibiotic therapy only in severe cases (WHO, Interim guidance March 13, 2020). It is becoming apparent that secondary bacterial infections occur in many COVID-19 patients and lead to increase disease severity and mortality, especially in those requiring invasive mechanical ventilation. Bacterial coinfection and secondary infection rates are generally low in COVID-19 patients, with higher rates in critically ill intensive care unit (ICU)-patients. Many studies of COVID-19 patients admitted to the ICU note the empiric use of antibiotics in a majority of patients, which can lead to increases in the prevalence of multidrug-resistant (MDR) bacteria (Contou et al., 2020; Sharifipour et al., 2020; Baskaran et al., 2021). COVID-19 pneumonia is associated with high rates of admission to the ICU and in-hospital mortality (Contou et al., 2020). Among COVID-19 patients, the bacteria, namely, Klebsiella pneumoniae, Acinetobacter spp., and Pseudomonas spp., Escherichia coli, and Staphylococcus spp., are the most frequently detected causative pathogens (Sharifipour et al., 2020). Understanding the proportion of COVID-19 patients with acute respiratory bacterial co-infection is crucial for treating patients with COVID-19 and to help ensure responsible use of antibiotics for decreased rates of antibiotic-resistant bacteria (Russell et al., 2021). We aimed to assess the rate of secondary bacterial infections, their antibiotic resistance, antibiotic therapies and clinical characteristics applied among patients admitted to our hospital for severe SARS-CoV-2 pneumonia in Isfahan, Iran.

\section{MATERIALS AND METHODS}

\section{Data Collection}

We conducted a single-center retrospective study including all adult ( $\geq 18$ years old) patients admitted to two COVID-19 ICUs from March 6 to September 7, 2020 in an academic hospital with approximately 220 beds, located in in Isfahan, Iran, for acute respiratory failure related to SARS-CoV-2 pneumonia. In this study, the criteria for admission to the ICU wards was a positive RT-PCR test or presence of ground glass opacity in the CT-scan. The collected clinical data include: age, gender, main symptoms, history of underlying disease, patient demographics, hospital stay, and outcomes and also the antibiotic and steroid regimen of the patient.

\section{Reverse Transcription Real-Time Polymerase Chain Reaction (RT-PCR) for the Detection of COVID-19}

This step was performed once for each patient. Briefly, nasopharyngeal and tracheal aspirate samples were taken from the patients and then placed in a separate collection tube containing 2-3 $\mathrm{ml}$ of viral transport medium and immediately sent to the COVID-19 reference laboratory of the university. RNA extraction was performed using the Viral Nucleic Acid Extraction kit according to the manufacturer's protocol (RBC Bioscience, Taipei, Taiwan). RT-PCR was performed using LightMix ${ }^{\circledR}$ Modular SARS and Wuhan CoV E-gene kit and using one-step RT-PCR polymerase Mix (Tib-Mol-biol, Berlin, Germany) (Corman VM et al., 2020).

\section{Collection of Clinical Specimens for Bacterial Detection}

In the present study, over six months, 365 clinical samples such as blood, catheters, cerebrospinal fluid (CSF), sputum, stool, tracheal aspirate, wound and urine were collected from inpatients in two different ICU wards of an educational hospital affiliated to the Isfahan University of Medical Sciences, Isfahan, Iran. The clinical samples were cultured on Blood agar, Chocolate Agar and MacConkey agar (Merck) and then incubated at $37^{\circ} \mathrm{C}$ for $24-48 \mathrm{~h}$ under standard conditions. Species identification was carried out using standard biochemical tests and API 20E (bioMérieux, Marcy-l'Etoile, France). In addition, Acinetobacter baumannii species were confirmed for bla OxA-51 gene by PCR (Turton et al., 2006).

\section{Antimicrobial Susceptibility Testing and MIC Determination}

Antimicrobial susceptibility testing was performed using the disc diffusion method according to the Clinical and Laboratory 
Standards Institute [CLSI] guidelines. The antibiotics tested included ceftazidime (CAZ), ceftriaxone (CTX), cefepime (CPM), ampicillin/sulbactam (SAM), piperacillin/tazobactam (PTZ), meropenem (MEM), amikacin (AM), gentamicin (GM), ciprofloxacin (CIP), and levofloxacin (LEV). The minimal inhibitory concentration (MIC) of meropenem was determined by gradient test strips (Liofilchem, Italy) based on the CLSI guideline (CLSI, 2017). MICs of colistin were determined by broth macrodilution method using colistin sulfate (SigmaAldrich), and EUCAST breakpoints were used for interpretation (EUCAST, 2017) (EUCAST). E. coli ATCC 25922 and S. aureus ATCC 29213 were used as quality control strains.

\section{Definitions}

A positive COVID-19 case was defined as a person with a confirmed positive result on a nasopharyngeal and tracheal swab tested using RT-PCR testing for SARS-CoV2 and also the presence of specific COVID-19 CT features. Secondary infections were identified by bacterial infection that developed during ICU stay but after admission of more than $48 \mathrm{~h}$ duration, meaning not present at the time of presentation with COVID-19. Secondary bacterial infections in COVID-19 patients are typically referred to as superinfections. Extensively drug resistant (XDR) was defined as non-susceptibility to at least one agent in all but two or fewer antimicrobial categories (i.e., bacterial isolates remain susceptible to only one or two available classes).

\section{Statistical Analysis}

Continuous data are described by median (range) and categorical data by absolute and relative frequencies. The categorical variables were denoted as absolute and relative values (frequencies) and compared by Fisher's exact Chi-square test using $\mathrm{P}<0.05$ as the level of significance. Statistical analyses were performed using Microsoft Excel 2007 and IBM SPSS Statistics Version 23.0.

\section{RESULTS}

During the study period, a total of 553 adult patients were admitted to our ICU wards (ICU-1 $\mathrm{N}=439$ and ICU-2 $\mathrm{N}=$ 114) for acute respiratory failure due to SARS-CoV-2 pneumonia. Bacterial and fungal positive culture results showed that sixty-five out of 553 patients (11.9\%) were positive for bacterial infections and six out of 553 of patients (1\%) had only fungal infections. As shown in Table 1, the median age of patients was 69.4 years (range of age 21-95 years), and 42 (63.6\%) patients were men. At least one underlying comorbidity was present in $53.8 \%$. Prevalent underlying diseases included heart disease $(21.5 \% ; \mathrm{n}=14)$ and diabetes mellitus (15.3\%; $\mathrm{n}=10$ ) (see Table 1). Approximately the median length of hospital stay and the median duration of ICU stay was 22 and 17 days, respectively. The overall mortality of the total population in both ICUs was $38.1 \%$ (211/ 553) $(\mathrm{ICU}-1=34.8 \%$ and ICU-2 $=50.8 \%)$. Mortality among
TABLE 1 | Baseline demographics and clinical characteristics of the study patients.

\begin{tabular}{|c|c|}
\hline Characteristics & Value $(n=65) \%$ \\
\hline Age median (mean \pm SD) & $69.4(21-95)$ \\
\hline \multicolumn{2}{|l|}{ Gender $\mathbf{n}(\%)$} \\
\hline Female & 24 (36.9\%) \\
\hline Male & $41(63.1 \%)$ \\
\hline \multicolumn{2}{|c|}{ Comorbidities no treatment for COVID-19*n (\%) } \\
\hline Diabetes & $10(15.3 \%)$ \\
\hline Hypertension & $3(4.6 \%)$ \\
\hline Chronic Kidney disease & $5(7.7 \%)$ \\
\hline COPD & $5(7.7 \%)$ \\
\hline Heart disease & $14(21.5 \%)$ \\
\hline Chronic neurological disease & $4(6.1 \%)$ \\
\hline Any medical immunosuppression & $3(4.6 \%)$ \\
\hline \multicolumn{2}{|c|}{ Symptom duration before admission, $\mathbf{n}(\%)$} \\
\hline Fever & $29(44.6 \%)$ \\
\hline Cough/shortness of breath & $58(89.2 \%)$ \\
\hline Muscular or joint pain & 27 (41.5\%) \\
\hline Nausea and vomiting & $8(12.3 \%)$ \\
\hline Anorexia & $20(30.7 \%)$ \\
\hline Headache & $5(7.7 \%)$ \\
\hline \multicolumn{2}{|c|}{ Radiology no treatment for COVID-19 } \\
\hline Ground-glass opacity (GGO) & $53(81.5 \%)$ \\
\hline Consolidation & $8(12.3 \%)$ \\
\hline Mix & $6(9.2 \%)$ \\
\hline \multicolumn{2}{|l|}{ COVID-19 treatment } \\
\hline Hydroxychloroquine & 25 (38.4\%) \\
\hline Oseltamivir & $3(4.6 \%)$ \\
\hline Kaletra & $2(3 \%)$ \\
\hline \multicolumn{2}{|l|}{ Outcomes } \\
\hline Mechanical ventilation & $40(61.5 \%)$ \\
\hline ICU length of stay (days) & $17(4-61)$ \\
\hline Hospitalization duration & $22(4-64)$ \\
\hline In-hospital mortality & $54(83.1 \%)$ \\
\hline Discharged & $11(16.9 \%)$ \\
\hline
\end{tabular}

${ }^{*}$ As defined by clinicians in admission records.

COVID-19 patients who acquired secondary bacterial infections was $83 \%$ (54/65), whereas mortality rate among patients without bacterial superinfections was $32.1 \%$ (211-54/488). There was a significantly higher rate of inpatient mortality ( $83 \%$ vs $32.1 \%$; $\mathrm{P}<.0001$ ) among patients with concomitant bacterial infection compared to those without. Also, fifty-six out of 65 received corticosteroids to the treatment of COVID19. Nineteen and 26 patients received dexamethasone $(6 \mathrm{mg} /$ day $)$ with a median duration of six (1-22) days and methylprednisolone $(2 \mathrm{mg} / \mathrm{kg} /$ day) with a median duration of four (1-8) days, respectively, and 11 patients received both (see Table 2 ).

In this study, of the 65 patients with secondary bacterial infection, 39 out of $65(60 \%)$ had positive RT-PCR results and 53 out of $65(81.5 \%)$ had a positive chest CT scan. Also, all 26 patients (40\%) with negative RT-PCR had typical appearance [ground glass opacity (GGO)] on chest CT. In addition, consolidation and mixed (GGO pulse consolidation) in chest CT scan of patients were $12.3 \%(8 / 65)$ and $9.2 \%(6 /$ 65), respectively.

Out of a total of 365 samples (279 samples in ICU-1 and 86 samples in ICU-2) collected for microbiological culture, 70 (19.2\%) and six (1.6\%) samples were positive for bacterial and fungal growth, respectively. Out of the total positive samples tested for bacteria, $62 / 70(88.6 \%)$ were respiratory specimens 
TABLE 2 | Characteristics of patients hospitalized with coronavirus disease 19 (COVID-19).

\begin{tabular}{|c|c|c|c|c|c|c|c|c|c|c|c|c|c|c|}
\hline Patient & Specimens & Species & Ward & $\begin{array}{c}\text { Date of } \\
\text { hospitalization }\end{array}$ & $\begin{array}{l}\text { Date of } \\
\text { intubation }\end{array}$ & $\begin{array}{l}\text { Sampling } \\
\text { date }\end{array}$ & $\begin{array}{l}\text { Length of stay in } \\
\text { ICU (Days) }\end{array}$ & $\begin{array}{l}\text { Hospitalization } \\
\text { duration }\end{array}$ & $\begin{array}{l}\text { RT- } \\
\text { PCR }\end{array}$ & $\begin{array}{c}\text { Antimicrobial } \\
\text { susceptibility phenotype }\end{array}$ & Outcomes & Comorbidities & $\begin{array}{c}\text { Treatment/ } \\
\text { duration (days)* }\end{array}$ & $\begin{array}{c}\text { Steroid/ } \\
\text { duration (days) }\end{array}$ \\
\hline 1. & Blood & A. baumannii & ICU-1 & $2020 / 3 / 15$ & $\mathrm{NI}$ & 2020/3/22 & 9 & 10 & Pos & SAM, COL & Died & COPD & $\mathrm{COL} / 3$ & MTP/4 \\
\hline 2. & $\begin{array}{l}\text { Sputum } \\
\text { Bal }\end{array}$ & $\begin{array}{l}\text { K. pneumoniae } \\
\text { K. pneumoniae }\end{array}$ & ICU-1 & 2020/3/9 & $\begin{array}{c}\mathrm{NI} \\
2020 / 5 / 3\end{array}$ & $\begin{array}{c}2020 / 3 / 28 \\
2020 / 5 / 6\end{array}$ & 61 & 64 & Pos & $\begin{array}{l}\mathrm{COL} \\
\mathrm{COL}\end{array}$ & Survived & $\begin{array}{l}\text { Diabetes, Heart } \\
\text { disease }\end{array}$ & COL/10 & MTP/6 \\
\hline 3. & TA & A. baumanniï & ICU-2 & $2020 / 3 / 24$ & 2020/3/25 & $2020 / 3 / 27$ & 7 & 15 & Neg & SAM, COL & Survived & - & COL/9 & $\mathrm{DXM} / 5$ \\
\hline 4. & TA & K. pneumoniae & ICU-1 & 2020/3/11 & 2020/3/19 & $2020 / 3 / 29$ & 23 & 22 & Pos & $\mathrm{COL}$ & Died & - & $\mathrm{COL} / 1$ & $\mathrm{DXM} / 5$ \\
\hline 5. & Sputum & A. baumannii & ICU-1 & $2020 / 3 / 15$ & $\mathrm{NI}$ & $2020 / 4 / 2$ & 22 & 24 & Pos & SAM, COL & Died & $\begin{array}{l}\text { Diabetes, Heart } \\
\text { disease }\end{array}$ & $\mathrm{COL} / 4$ & $\mathrm{DXM} / 5, \mathrm{MTP} / 2$ \\
\hline 6. & TA & K. pneumoniae & ICU-1 & 2020/3/14 & 2020/3/20 & 2020/4/2 & 26 & 27 & $\mathrm{Neg}$ & $\mathrm{COL}$ & Died & - & COL/5 & MTP/8 \\
\hline 7. & TA & K. pneumoniae & ICU-1 & $2020 / 3 / 25$ & $2020 / 4 / 3$ & 2020/4/5 & 38 & 41 & Neg & $\mathrm{AM}, \mathrm{COL}$ & Died & Heart disease & COL/3 & DXM/2, MTP/4 \\
\hline 8. & TA & A. baumannii & ICU-1 & $2020 / 3 / 27$ & 2020/3/28 & 2020/4/5 & 32 & 45 & Pos & SAM, COL & Survived & - & $\mathrm{COL} / 4$ & DXM/2, MTP/6 \\
\hline 9. & Sputum & K. pneumoniae & ICU-1 & $2020 / 3 / 25$ & $\mathrm{NI}$ & $2020 / 4 / 5$ & 9 & 12 & Pos & $\mathrm{COL}$ & Died & - & - & $\mathrm{DXM} / 5$ \\
\hline 10. & TA & A. baumannii & ICU-2 & $2020 / 3 / 18$ & 2020/3/30 & 2020/4/11 & 23 & 25 & Pos & $\mathrm{COL}$ & Died & - & $\mathrm{COL} / 1$ & DXM/6, MTP/2 \\
\hline 11. & TA & A. baumannii & ICU-1 & $2020 / 4 / 11$ & $2020 / 4 / 17$ & $2020 / 4 / 20$ & 10 & 11 & Pos & COL & Died & - & - & MTP/3 \\
\hline 12. & $\begin{array}{l}\text { TA } \\
\text { Wound }\end{array}$ & $\begin{array}{l}\text { A. baumannii } \\
\text { K. pneumoniae }\end{array}$ & ICU-1 & $2020 / 4 / 17$ & $2020 / 4 / 19$ & $\begin{array}{l}2020 / 4 / 22 \\
2020 / 4 / 25\end{array}$ & 30 & 31 & Pos & $\begin{array}{l}\mathrm{COL} \\
\mathrm{COL}\end{array}$ & Died & - & $\mathrm{COL} / 4$ & $\mathrm{DXM} / 22$ \\
\hline 13. & TA & A. baumannii & ICU-1 & $2020 / 4 / 17$ & $2020 / 4 / 25$ & $2020 / 4 / 25$ & 7 & 8 & Neg & COL & Died & - & - & - \\
\hline 14. & TA & A. baumannii & ICU-1 & $2020 / 3 / 25$ & 2020/4/3 & $2020 / 4 / 30$ & 35 & 41 & Neg & SAM, COL & Died & $\begin{array}{l}\text { Diabetes, Heart } \\
\text { disease }\end{array}$ & $\mathrm{COL} / 3$ & $\mathrm{DXM} / 2, \mathrm{MTP} / 3$ \\
\hline 15. & Sputum & K. pneumoniae & ICU-1 & 2020/5/5 & $\mathrm{NI}$ & $2020 / 5 / 10$ & 8 & 9 & Neg & $\mathrm{COL}$ & Died & & $\mathrm{COL} / 1$ & $\mathrm{DXM} / 2$ \\
\hline 16. & Blood & E. coli & ICU-1 & $2020 / 4 / 30$ & $\mathrm{NI}$ & 2020/5/12 & 11 & 12 & Pos & AM, PTZ, CAZ, MEM, COL, & Died & $\begin{array}{l}\text { CKD, COPD, } \\
\text { Heart disease }\end{array}$ & CAZ/10, COL/1 & - \\
\hline 17. & Urine & A. baumannii & ICU-1 & $2020 / 5 / 4$ & $\mathrm{NI}$ & 2020/5/16 & 20 & 22 & Neg & $\mathrm{COL}$ & Died & - & $\mathrm{COL} / 1$ & $\mathrm{DXM} / 3$ \\
\hline 18. & TA & A. baumannii & ICU-1 & 2020/5/2 & 2020/5/2 & 2020/5/17 & 25 & 56 & Neg & $\mathrm{COL}$ & Survived & Hypertension & COL/34 & $\mathrm{DXM} / 1$ \\
\hline 19. & TA & K. pneumoniae & ICU-1 & $2020 / 3 / 27$ & 2020/3/27 & 2020/4/17 & 30 & 31 & Neg & COL & Died & - & - & $\mathrm{DXM} / 1$ \\
\hline 20. & $\begin{array}{l}\text { TA } \\
\text { Blood }\end{array}$ & $\begin{array}{l}\text { K. pneumoniae } \\
\text { K. pneumoniae }\end{array}$ & ICU-1 & 2020/5/14 & 2020/5/15 & $2020 / 5 / 21$ & 10 & 11 & Pos & $\begin{array}{l}\text { AM, GM, MEM, COL } \\
\text { AM, COL }\end{array}$ & Died & Heart disease & $\mathrm{MEM} / 3, \mathrm{COL} / 1$ & $\mathrm{DXM} / 5$ \\
\hline 21. & Sputum & $\begin{array}{l}\text { A. baumannii } \\
\text { K. pneumoniae }\end{array}$ & ICU-1 & $2020 / 5 / 11$ & $\mathrm{NI}$ & $2020 / 5 / 21$ & 4 & 10 & Pos & $\begin{array}{l}\mathrm{AM}, \mathrm{COL} \\
\mathrm{AM}, \mathrm{COL}\end{array}$ & Died & Heart disease & - & MTP/3 \\
\hline 22. & Blood & K. pneumoniae & ICU-1 & 2020/5/4 & $\mathrm{NI}$ & $2020 / 5 / 23$ & 21 & 32 & Neg & $\mathrm{AM}, \mathrm{COL}$ & Died & - & $\mathrm{COL} / 1$ & $\mathrm{DXM} / 3$ \\
\hline 23. & TA & K. pneumoniae & ICU-1 & $2020 / 5 / 21$ & $2020 / 5 / 21$ & $2020 / 5 / 24$ & 16 & 17 & Neg & $\mathrm{AM}, \mathrm{GM}, \mathrm{COL}$ & Died & CND & $\mathrm{COL} / 7$ & MTP/1 \\
\hline 24. & Sputum & $\begin{array}{l}\text { A. baumannii } \\
\text { K. pneumoniae }\end{array}$ & ICU-1 & $2020 / 5 / 10$ & $\mathrm{NI}$ & $2020 / 5 / 27$ & 30 & 31 & Pos & $\begin{array}{l}\mathrm{COL} \\
\mathrm{COL}\end{array}$ & Died & - & $\mathrm{COL} / 14$ & $\mathrm{DXM} / 10$ \\
\hline 25. & TA & A. baumannii & ICU-1 & 2020/5/11 & 2020/5/25 & $2020 / 5 / 27$ & 6 & 16 & Pos & SAM, COL & Died & $\begin{array}{l}\text { COPD, Heart } \\
\text { disease }\end{array}$ & - & MTP/4 \\
\hline 26. & Sputum & A. baumanniï & ICU-1 & $2020 / 5 / 16$ & $\mathrm{NI}$ & $2020 / 5 / 27$ & 11 & 20 & Pos & SAM, COL & Died & - & COL/5 & - \\
\hline 27. & TA & $\begin{array}{l}\text { A. baumannii } \\
\text { K. pneumoniae }\end{array}$ & ICU-1 & $2020 / 5 / 15$ & $2020 / 5 / 20$ & $2020 / 5 / 30$ & 16 & 17 & Neg & $\begin{array}{l}\mathrm{COL} \\
\mathrm{COL}\end{array}$ & Died & Hypertension & $\mathrm{COL} / 2$ & - \\
\hline 28. & Sputum & A. baumannii & ICU-1 & $2020 / 5 / 20$ & $\mathrm{NI}$ & 2020/6/3 & 9 & 17 & Neg & $\mathrm{COL}$ & Died & Heart disease & $\mathrm{COL} / 3$ & MTP/3 \\
\hline 29. & TA & $\begin{array}{l}\text { A. baumannii } \\
\text { K. pneumoniae }\end{array}$ & ICU-1 & $2020 / 5 / 31$ & $2020 / 5 / 31$ & $2020 / 6 / 5$ & 6 & 7 & Pos & $\begin{array}{l}\mathrm{COL} \\
\mathrm{COL}\end{array}$ & Died & Hypertension & - & MTP/4 \\
\hline 30. & TA & $\begin{array}{l}\text { A. baumannii } \\
\text { K. pneumoniae }\end{array}$ & $\mathrm{ICU}-2$ & 2020/5/2 & 2020/5/2 & 2020/5/6 & 21 & 53 & Neg & $\begin{array}{l}\mathrm{COL} \\
\mathrm{COL}\end{array}$ & Survived & CND & COL/27 & $\mathrm{DXM} / 3$ \\
\hline 31. & Sputum & A. baumannii & ICU-1 & $2020 / 5 / 30$ & $\mathrm{NI}$ & 2020/6/9 & 10 & 11 & Pos & SAM, COL & Died & Heart disease & - & MTP/2 \\
\hline 32. & TA & A. baumannii & ICU-1 & 2020/6/2 & 2020/6/9 & 2020/6/11 & 9 & 10 & Pos & SAM, COL & Died & - & - & MTP/4 \\
\hline 33. & Sputum & A. baumannii & ICU-1 & 2020/6/9 & $\mathrm{NI}$ & 2020/6/12 & 3 & 4 & Neg & $\mathrm{AM}, \mathrm{GM}$ & Died & - & - & - \\
\hline 34. & Sputum & $\begin{array}{l}\text { A. baumannii } \\
\text { K. pneumoniae }\end{array}$ & ICU-1 & $2020 / 5 / 19$ & $\mathrm{NI}$ & $2020 / 6 / 13$ & 24 & 25 & Pos & $\begin{array}{l}\mathrm{COL} \\
\mathrm{COL}\end{array}$ & Died & CND & $\mathrm{COL} / 1$ & $\mathrm{DXM} / 2, \mathrm{MTP} / 2$ \\
\hline 35. & TA & K. pneumoniae & $\mathrm{ICU}-2$ & 2020/6/8 & 2020/6/14 & 2020/6/17 & 12 & 14 & Pos & COL & Died & - & $\mathrm{COL} / 4$ & MTP/5 \\
\hline 36. & TA & P. aeruginosa & ICU-2 & 2020/6/3 & 2020/6/15 & 2020/6/19 & 20 & 30 & Neg & AM, GM, PTZ, MEM, COL & Died & - & $\mathrm{MEM} / 12$ & - \\
\hline 37. & Blood & S. epidermidis & ICU-1 & $2020 / 5 / 2$ & $\mathrm{NI}$ & 2020/6/22 & 20 & 50 & Neg & VAN, LEN, TEC & Survived & CND & COL/22, LIN/14 & $\mathrm{DXM} / 1$ \\
\hline 38. & Sputum & K. pneumoniae & $\mathrm{ICU}-2$ & 2020/6/7 & $\mathrm{NI}$ & $2020 / 6 / 24$ & 18 & 20 & Pos & - & Died & - & $\mathrm{COL} / 4$ & MTP/7 \\
\hline 39. & TA & K. pneumoniae & ICU-1 & $2020 / 6 / 10$ & 2020/6/15 & $2020 / 6 / 25$ & 14 & 15 & Pos & COL & Died & & - & MTP/5 \\
\hline 40. & Sputum & A. baumannii & ICU-1 & 2020/6/8 & $\mathrm{NI}$ & $2020 / 6 / 27$ & 27 & 28 & Neg & $\mathrm{COL}$ & Died & MI & $\mathrm{COL} / 14$ & MTP/3 \\
\hline
\end{tabular}




\begin{tabular}{|c|c|c|c|c|c|c|c|c|c|c|c|c|c|c|}
\hline Patient & Specimens & Species & Ward & $\begin{array}{c}\text { Date of } \\
\text { hospitalization }\end{array}$ & $\begin{array}{c}\text { Date of } \\
\text { intubation }\end{array}$ & $\begin{array}{l}\text { Sampling } \\
\text { date }\end{array}$ & $\begin{array}{l}\text { Length of stay in } \\
\text { ICU (Days) }\end{array}$ & $\begin{array}{l}\text { Hospitalization } \\
\text { duration }\end{array}$ & $\begin{array}{l}\text { RT- } \\
\text { PCR }\end{array}$ & $\begin{array}{l}\text { Antimicrobial } \\
\text { susceptibility phenotype }\end{array}$ & Outcomes & Comorbidities & $\begin{array}{c}\text { Treatment/ } \\
\text { duration (days)* }\end{array}$ & $\begin{array}{c}\text { Steroid/ } \\
\text { duration (days) }\end{array}$ \\
\hline 41. & TA & $\begin{array}{l}\text { K. pneumoniae } \\
\text { K. pneumoniae }\end{array}$ & ICU-1 & 2020/6/20 & 2020/6/28 & 2020/7/1 & 11 & 13 & Neg & $\begin{array}{l}\mathrm{COL} \\
\mathrm{COL}\end{array}$ & Died & COPD, MI & - & $\begin{array}{l}\mathrm{DXM} / 5 \text {, } \\
\mathrm{MTP} / 3\end{array}$ \\
\hline 42. & Sputum & $\begin{array}{l}\text { A. baumannii } \\
\text { K. pneumoniae }\end{array}$ & ICU-1 & 2020/6/23 & $\mathrm{NI}$ & 2020/7/1 & 10 & 11 & Neg & $\begin{array}{l}\mathrm{COL} \\
\mathrm{COL}\end{array}$ & Died & Heart disease & - & - \\
\hline 43. & Sputum & K. pneumoniae & ICU-1 & 2020/6/10 & $\mathrm{NI}$ & 2020/6/25 & 14 & 15 & Pos & COL & Died & Diabetes & - & MTP/5 \\
\hline 44. & Sputum & K. pneumoniae & ICU-1 & 2020/6/23 & $\mathrm{NI}$ & 2020/7/5 & 16 & 26 & Pos & COL & Survived & - & COL/10 & MTP/5 \\
\hline 45. & TA & $\begin{array}{l}\text { A. baumannii } \\
\text { K. pneumoniae } \\
\text { K. pneumoniae }\end{array}$ & ICU-1 & 2020/6/17 & 2020/6/28 & $\begin{array}{l}2020 / 7 / 7 \\
2020 / 7 / 27\end{array}$ & 51 & 53 & Pos & $\begin{array}{l}\mathrm{COL} \\
\mathrm{COL}\end{array}$ & Died & CKD & $\mathrm{COL} / 18, \mathrm{TIG} / 12$ & $\mathrm{DXM} / 22$ \\
\hline 46. & $\begin{array}{l}\text { Bal } \\
\text { TA }\end{array}$ & $\begin{array}{l}\text { K. pneumoniae } \\
\text { K. pneumoniae }\end{array}$ & ICU-1 & 2020/6/13 & 2020/6/21 & $2020 / 6 / 27$ & 23 & 28 & Neg & $\mathrm{COL}$ & Died & - & $\mathrm{COL} / 10$ & $\mathrm{DXM} / 5, \mathrm{MTP} / 4$ \\
\hline 47. & Sputum & $\begin{array}{l}\text { A. baumannii } \\
\text { K. pneumoniae }\end{array}$ & ICU-1 & 2020/6/18 & $\mathrm{NI}$ & 2020/6/27 & 13 & 14 & Neg & $\begin{array}{l}\mathrm{COL} \\
\mathrm{COL}\end{array}$ & Survived & - & $\mathrm{COL} / 6$ & $\mathrm{DXM} / 4$ \\
\hline 48. & TA & $\begin{array}{l}\text { A. baumannii } \\
\text { K. pneumoniae }\end{array}$ & ICU-1 & 2020/7/11 & 2020/7/11 & 2020/7/18 & 23 & 24 & Neg & $\begin{array}{l}\mathrm{COL} \\
\mathrm{COL}\end{array}$ & Died & - & COL/18 & - \\
\hline 49. & Sputum & $\begin{array}{l}\text { A. baumannii } \\
\text { K. pneumoniae }\end{array}$ & ICU-2 & 2020/7/9 & $\mathrm{NI}$ & $2020 / 7 / 20$ & 7 & 11 & Pos & $\begin{array}{l}\text { AM, COL } \\
\text { COL }\end{array}$ & Died & CKD, COPD & - & MTP/7 \\
\hline 50. & TA & $\begin{array}{l}\text { A. baumannii } \\
\text { K. pneumoniae }\end{array}$ & ICU-2 & 2020/7/22 & 2020/8/5 & 2020/8/5 & 13 & 14 & Pos & $\begin{array}{l}\mathrm{COL} \\
\mathrm{COL}\end{array}$ & Died & Heart disease & - & MTP/3 \\
\hline 51. & TA & K. pneumoniae & ICU-1 & $2020 / 7 / 30$ & 2020/8/3 & 2020/8/5 & 9 & 10 & Pos & $\mathrm{COL}$ & Died & CKD & COL/6 & DXM/3, MTP/3 \\
\hline 52. & TA & $\begin{array}{l}\text { A. baumannii } \\
\text { K. pneumoniae }\end{array}$ & ICU-1 & $2020 / 7 / 28$ & 2020/8/1 & 2020/8/7 & 10 & 12 & Pos & $\begin{array}{l}\mathrm{COL} \\
\mathrm{COL}\end{array}$ & Died & Diabetes & - & MTP/4 \\
\hline 53. & Sputum & K. pneumoniae & ICU-1 & $2020 / 7 / 17$ & $\mathrm{Nl}$ & 2020/8/8 & 25 & 24 & Pos & $\begin{array}{l}\text { AM, GM, PTZ, SAM, CAZ, } \\
\text { LEV, MEM, COL, }\end{array}$ & Died & Ml & $\begin{array}{l}\mathrm{MEM} / 7, \mathrm{LEV} / 7 \\
\mathrm{COL} / 13\end{array}$ & $\mathrm{DXM} / 8$ \\
\hline 54. & TA & A. baumannii & ICU-1 & 2020/7/31 & $2020 / 7 / 31$ & 2020/8/9 & 30 & 33 & Neg & SAM, COL & Survived & - & COL/16 & $\mathrm{DXM} / 18$ \\
\hline 55. & $\begin{array}{l}\text { TA } \\
\text { TA }\end{array}$ & $\begin{array}{l}\text { A. baumannii } \\
\text { Enterobacter }\end{array}$ & ICU-1 & $2020 / 7 / 27$ & $2020 / 8 / 4$ & $\begin{array}{c}2020 / 8 / 7 \\
2020 / 8 / 13\end{array}$ & 20 & 21 & Pos & $\begin{array}{l}\text { COL } \\
\text { PTZ, CAZ }\end{array}$ & Died & - & $\mathrm{COL} / 7$ & MTP/4 \\
\hline 56. & TA & $\begin{array}{l}\text { A. baumannii } \\
\text { K. pneumoniae }\end{array}$ & ICU-2 & 2020/8/13 & $2020 / 8 / 18$ & 2020/8/20 & 6 & 7 & Neg & $\begin{array}{l}\text { SAM, COL } \\
\text { COL }\end{array}$ & Died & - & - & MTP/2 \\
\hline 57. & Blood & K. pneumoniae & ICU-1 & $2020 / 7 / 29$ & $\mathrm{NI}$ & $2020 / 8 / 21$ & 22 & 23 & Pos & COL & Died & - & COL/10 & MTP/5 \\
\hline 58. & TA & K. pneumoniae & ICU-1 & 2020/8/6 & 2020/8/18 & 2020/8/21 & 13 & 16 & Pos & - & Died & $\begin{array}{l}\text { Diabetes, Heart } \\
\text { disease }\end{array}$ & $\mathrm{COL} / 2$ & MTP/8 \\
\hline 59. & TA & K. pneumoniae & ICU-2 & 2020/8/18 & 2020/8/21 & 2020/8/24 & 2 & 6 & Pos & - & Died & - & - & - \\
\hline 60. & TA & K. pneumoniae & ICU-1 & 2020/8/19 & 2020/8/19 & $2020 / 8 / 24$ & 10 & 22 & Neg & CIP, LEV, COL & Survived & - & COL/14 & $\mathrm{DXM} / 8$ \\
\hline 61. & TA & K. pneumoniae & ICU-2 & 2020/8/14 & 2020/8/16 & 2020/8/25 & 16 & 17 & Pos & COL & Died & - & $\mathrm{COL} / 4$ & DXM/2, MTP/4 \\
\hline 62. & TA & K. pneumoniae & ICU-1 & 2020/8/22 & $2020 / 8 / 27$ & 2020/8/27 & 10 & 20 & Pos & COL & Survived & Diabetes & COL/12 & $\mathrm{DXM} / 10$ \\
\hline 63. & TA & K. pneumoniae & ICU-1 & 2020/8/19 & $2020 / 8 / 19$ & $2020 / 8 / 28$ & 13 & 15 & Pos & COL & Died & Diabetes & $\mathrm{COL} / 3$ & MTP/2 \\
\hline 64. & Sputum & K. pneumoniae & ICU-1 & $2020 / 8 / 10$ & $\mathrm{NI}$ & 2020/8/31 & 19 & 22 & Pos & $\mathrm{COL}$ & Died & $\begin{array}{l}\text { Diabetes, Heart } \\
\text { disease }\end{array}$ & $\mathrm{COL} / 4$ & MTP/6 \\
\hline 65. & TA & $\begin{array}{l}\text { A. baumannii } \\
\text { K. pneumoniae }\end{array}$ & ICU-1 & 2020/7/23 & 2020/8/27 & 2020/9/6 & 20 & 45 & Pos & $\begin{array}{l}\text { SAM, COL } \\
\text { COL }\end{array}$ & Died & Diabetes, CKD & COL/14 & DXM/15, MTP/4 \\
\hline
\end{tabular}

Bal, Bronchoalveolar lavage; TA, Tracheal Aspirate; ICU, Intensive Care Unit; NI, Not Intubate; Pos, Positive; Neg, Negative; SAM, Ampicillin/sulbactam; COL, Colistin; AM, Amikacin; PTZ, Piperacillin-tazobactam; CAZ, Ceftazidime; MEM, Meropenem; GM, Gentamicin; VAN, Vancomycin; LIN, Linezolid; TEC, Teicoplanin; CIP, Ciprofloxacin; LEV, Levofloxacin; TIG, Tigecycline; CKD, Chronic Kidney disease; COPD, Chronic obstructive pulmonary disease; CND, Chronic neurological disease; MI, Medical immunosuppression; MTP, Methyl-prednisolone; DXM, Dexamethasone.

*In this column, only the antibiotics prescribed after the diagnosis of bacterial infections are listed. 
[endotracheal aspirate, sputum and bronchoalveolar lavage (BAL) ], 6/70 (8.6\%) were blood samples, $1 / 70(1.4 \%)$ was a urine sample and 1/70 (1.4\%) was a wound sample. Overall, 86 bacterial isolates were collected from 65 patients. Seventy-two strains (83.7\%) were recovered from 54 patients in ICU-1, whereas the remaining isolates were recovered from 11 inpatients in ICU-2 ward. Also, out of six positive samples for fungal isolates, six Candida species were reported and all of them were collected from urine samples. According to culture results, the commonest co-pathogens identified were mainly Gramnegative bacteria (GNB), including K. pneumoniae $(\mathrm{n}=47), A$. baumannii $(\mathrm{n}=35)$, Enterobacter cloacae $(\mathrm{n}=1)$, E. coli $(\mathrm{n}=1)$, and Pseudomonas aeruginosa $(\mathrm{n}=1)$. Only one Gram-positive bacteria (S. epidermidis) was isolated. Mixed infection with more than one bacteria isolated from the same site was seen in $24.6 \%$ $(16 / 65)$ of patients. The results are shown in Table 2 . In this study, pneumonia was the most commonly reported bacterial infection $(n=62)$, followed by bacterial bloodstream infection $(n=6)$, bacterial wound infection $(n=1)$ and bacterial urinary tract infection $(n=1)$. It should be noted that some patients develop more than one infection during hospitalization in the ICU wards (see Table 2). Also, six fungal urinary tract infections caused by Candida species were observed.

In this study, thirty-eight patients were diagnosed with ventilator-associated pneumonia (VAP). Eighteen patients were early-onset VAP (EOVAP) and 20 patients were late-onset VAP (LOVAP) (see Table 2).

\section{Antimicrobial Susceptibility}

Susceptibility profiles against eleven antimicrobials agents are listed in Table 3. Highest resistance was seen in K. pneumoniae isolates against nearly all antimicrobials agents. The rate of $K$. pneumoniae isolates exhibiting resistance to colistin was $10.6 \%$ (5/47), with MICs ranging from 4 to $16 \mathrm{mg} / \mathrm{L}$. All A. baumannii isolates demonstrated resistance to meropenem, ceftazidime, cefotaxime, cefepime, piperacillin/tazobactam, fluoroquinolones and all of them were susceptible to colistin. The percentages of resistance to other antimicrobial agents among $A$. baumannii isolates were as follows: amikacin 94.3\% (33/35); gentamicin $97.2 \%(34 / 35)$ and ampicillin/sulbactam 60\% (21/35).
S. epidermidis were resistant to all antimicrobial agents, with the exception of vancomycin, linezolid, and teicoplanin.

\section{Patterns of Antibiotic Use and Antiviral Therapy}

Thirty-six of the $65(55.4 \%)$ of patients received antimicrobial therapy within $24 \mathrm{~h}$ of admission: in twenty-five patients antimicrobial therapy was initiated in the emergency department, and in 11 patients therapy was initiated on ICU (in cases of direct admittance to ICU). In terms of antibiotic prescribing patterns, $100 \%(n=65)$ of all patients received at least two antibiotics. The most frequently used antibiotic regimen was meropenem $(56 / 6586.2 \%)$ with a median duration of 12 (232) days, followed by levofloxacin (48/65 70.8\%), colistin (44/65 $67.7 \%)$, linezolid (40/65 61.5\%), vancomycin (31/65 47.7\%), with a median duration of 9 (2-24), 8 (1-34), 10 (2-14) and 7 (2-14) days, respectively. Detailed information on antibiotic therapy including duration of therapy is presented in Table 4. Also, among 65 patients with superinfection, 38.4, 4.6, and $3 \%$ patients received hydroxychloroquine, oseltamivir, and kaletra, respectively (Table $\mathbf{1}$ ).

\section{DISCUSSION}

This study belongs to a group of early microbiological studies that report clinical characteristics and outcomes in COVID-19 patients admitted to the ICUs with secondary bacterial infections in Iran. The main results are as follows: (1) 11.9\% of the COVID19 patients admitted to our ICUs have a superinfection upon ICU admission; (2) the leading involved bacteria were extremely drug-resistant (XDR) GNB specially $K$. pneumoniae and $A$. baumannii; (3) inappropriate use or overuse of antibiotics and change in antimicrobial use during the pandemic. At present, there are limited data regarding bacterial co-infection and secondary infection in COVID-19 in Iran. However, in a study carried out by Sharifipour et al. in Qom, Iran, it was reported that bacterial co-infection with $A$. baumannii and Staphylococcus aureus identified in nineteen COVID-19 patients admitted to the ICU (Sharifipour et al., 2020). It is important to mention that

TABLE 3 | Drug resistance rate of five GNB to antibiotics in patients with COVID-19 from January to April in Isfahan, Iran.

\begin{tabular}{|c|c|c|c|c|c|c|c|c|c|c|c|c|c|c|c|}
\hline \multirow[t]{2}{*}{ Antibiotics } & \multicolumn{3}{|c|}{ K. pneumoniae (47) } & \multicolumn{3}{|c|}{ A. baumannii (35) } & \multicolumn{3}{|c|}{ E. coli (1) } & \multicolumn{3}{|c|}{ P. aeruginosa (1) } & \multicolumn{3}{|c|}{ Enterobacter spp. (1) } \\
\hline & $\mathbf{S} \%$ & $1 \%$ & $\mathbf{R} \%$ & $\mathbf{S} \%$ & $1 \%$ & $\mathbf{R} \%$ & S\% & $1 \%$ & $\mathbf{R} \%$ & $\mathbf{S} \%$ & $1 \%$ & $\mathbf{R} \%$ & $\mathbf{S} \%$ & $1 \%$ & $\mathbf{R} \%$ \\
\hline Ceftazidime & 2.1 & 0 & 97.9 & 0 & 0 & 100 & 100 & 0 & 0 & 0 & 0 & 100 & 100 & 0 & 0 \\
\hline Ceftriaxone & 2.1 & 0 & 97.9 & 0 & 0 & 100 & 0 & 0 & 100 & 0 & 0 & 100 & 0 & 0 & 100 \\
\hline Cefepime & 2.1 & 0 & 97.9 & 0 & 0 & 100 & 100 & 0 & 0 & 100 & 0 & 0 & 100 & 0 & 0 \\
\hline Ampicillin/sulbactam & 2.1 & 0 & 97.9 & 34.3 & 5.7 & 60 & 0 & 0 & 100 & 0 & 0 & 100 & 0 & 0 & 100 \\
\hline Piperacillin/tazobactam & 2.1 & 0 & 97.9 & 0 & 0 & 100 & 100 & 0 & 0 & 100 & 0 & 0 & 100 & 0 & 0 \\
\hline Meropenem & 4.2 & 0 & 95.8 & 0 & 0 & 100 & 100 & 0 & 0 & 100 & 0 & 0 & 0 & 0 & 100 \\
\hline Amikacin & 17 & 2.1 & 80.9 & 5.7 & 0 & 94.3 & 100 & 0 & 0 & 100 & 0 & 0 & 0 & 0 & 100 \\
\hline Gentamicin & 6.4 & 0 & 93.6 & 2.8 & 0 & 97.2 & 100 & 0 & 0 & 100 & 0 & 0 & 0 & 0 & 100 \\
\hline Ciprofloxacin & 2.1 & 0 & 97.9 & 0 & 0 & 100 & 0 & 0 & 100 & 0 & 0 & 100 & 0 & 0 & 100 \\
\hline Levofloxacin & 4.2 & 0 & 95.8 & 0 & 0 & 100 & 0 & 0 & 100 & 0 & 0 & 100 & 0 & 0 & 100 \\
\hline Colistin & 89.4 & 0 & 10.6 & 100 & 0 & 0 & 100 & 0 & 0 & 100 & 0 & 0 & 0 & 0 & 100 \\
\hline
\end{tabular}


TABLE 4 | Most commonly used antibiotics for treatment of patients with secondary bacterial infection.

\begin{tabular}{lc}
\hline Use of empirical antibiotics, $\mathbf{n}(\%)$ & Value (n = 65) \\
\hline Use of more than one class of empirical antibiotics, n (\%) & $65(100 \%)$ \\
Cephalosporin (Ceftazidime, Ceftriaxone), n (\%) & $13(20 \%)$ \\
Duration of therapy (days) & $7(2-15)$ \\
Azithromycin, n (\%) & $7(10.7 \%)$ \\
Duration of therapy (days) & $4(3-6)$ \\
Aminoglycosides (Amikacin, Tobramycin), n (\%) & $4(6 \%)$ \\
Duration of therapy (days) & $7(5-10)$ \\
Levofloxacin, $n$ (\%) & $48(73.8 \%)$ \\
Duration of therapy (days) & $9(2-24)$ \\
Piperacillin/tazobactam, $n(\%)$ & $14(21.5 \%)$ \\
Duration of therapy (days) & $8(3-12)$ \\
Ampicillin/sulbactam, $n(\%)$ & $5(7.7 \%)$ \\
Duration of therapy (days) & $8(4-14)$ \\
Meropenem, $n$ (\%) & $56(86.2 \%)$ \\
Duration of therapy (days) & $12(2-32)$ \\
Colistin, $n$ (\%) & $44(67.7 \%)$ \\
Duration of therapy (days) & $8(1-34)$ \\
Vancomycin, n (\%) & $31(47.7 \%)$ \\
Duration of therapy (days) & $7(2-14)$ \\
Linezolid, $n$ (\%) & $40(61.5 \%)$ \\
Duration of therapy (days) & $10(2-14)$ \\
\hline
\end{tabular}

all data collected and analyzed in this study is related to pre tocilizumab era.

The prevalence of bacterial infections in COVID-19 patients admitted to the ICU has been reported in several studies. (Contou et al., 2020; Sharifipour et al., 2020; Baskaran et al., 2021). A recent systematic review and meta-analysis study evaluating co-infections among patients infected with COVID19 performed by Langford et al. reported that the rate of secondary bacterial infection is $14.3 \%$ (Langford et al., 2020). Recent studies reported 41 and $28 \%$ rates of co-infection among patients admitted to a North American and French ICUs (Contou et al., 2020; Lehmann et al., 2020). Respiratory sites were the most common sites of bacterial infection in COVID-19 patients. Gram-negative pathogens were predominant in respiratory infections. Herein, we report on a $11.9 \%$ rate of bacterial superinfection mostly due to carbapenem resistant $K$. pneumoniae (CRKP) and A. baumannii (CRAB). This observation was similar to the findings reported by previous studies that investigated bacterial co-infections in patients with COVID-19, particularly ICU cohorts (Contou et al., 2020; Sharifipour et al., 2020; Baskaran et al., 2021). In this COVID19 pandemic, Yang et al. have reported that bacterial coinfections were found in around 11.5\% COVID-19 patients, which is in accordance with our results (11.9\%) (Yang et al., 2020). According to recent studies in Iran, CRKP and CRAB had the highest rates of incidence in ICUs (Solgi et al., 2017; Sharifipour et al., 2020; Solgi et al., 2020; Bolourchi et al., 2021; Nazari et al., 2021).

During the study period, 38 patients were diagnosed with VAP. Eighteen patients were EOVAP and 20 patients were LOVAP (see Table 2). Pathogens from this study did not differ between EOVAP and LOVAP. The results of our study were similar to other tertiary centers in India and Thailand (Arayasukawat et al., 2012; Jakribettu et al., 2016). In addition, we observed 6 cases of fungal co-infections caused by Candida species. In a systematic review in 2020, Lansbury and colleagues have reported that the rate of fungal infections was low among COVID-19 patients, which is in line with our results (Lansbury et al., 2020).

The median length of ICU stay among patients in our study was high, 17 days (4 to 61), findings similar to the study of Sharifipour et al. (2020), which reported a length of stay of 15 days (2-39) of all patients with COVID-19 admitted to their ICU. In this study, there was a significantly higher rate of inpatient mortality ( $83 \%$ 54/65) among patients with secondary bacterial infection compared to those without superinfection $(211-54 / 488=32.1 \%)$. Among patients admitted to ICU wards, we found an association between patients identified to have superinfection and inpatient mortality on univariable analysis $(\mathrm{P}<.0001)$. In a study conducted by Neto and colleagues on COVID-19 patients with secondary bacterial infections in the United States, mortality rate was 50\% (OR, 5.838; P <.0001) (Neto et al., 2020). Similarly, the study by Vijay and colleagues in COVID-19 cases indicated that the mortality among patients who acquired secondary infections was $56.7 \%$ (Vijay et al., 2021).

Despite an overall low rate of bacterial infections in our study, all of 65 patients who acquired bacterial infection received at least two empiric antibiotic therapy upon ICU admission before first positive MDR-GNB culture, with the majority constituting broadspectrum agents such as levofloxacin, meropenem and linezolid, which can cause the lack of growth of not-MDR pathogens in culture samples. Similarly, the study by Patel and colleagues in COVID-19 cases indicated that of the 71 patients who had MDR GNB infection, 69 (97\%) had received antibiotics before first positive resistant GNB culture (Patel et al., 2021). Moreover, these findings are similar to a study in New York reported empirical antibiotics use among 79\% of COVID-19 inpatients (Nori et al., 2020) and several studies performed during the beginning of the COVID-19 pandemic from China have reported that over $90 \%$ of patients received empirical antibiotics (Wang et al., 2020; Wu et al., 2020). In the present study, GNB were the most commonly recovered organisms from specimen cultures in patients with COVID-19. The predominance of GNB in this study likely reflects nosocomial infection following prolonged ICU stay and empirical antibiotic use.

The frequent prescription of broad-spectrum empirical antimicrobials in COVID-19 patients could increase antibiotic resistance in the near future. Therefore, strict programs of antimicrobial stewardship are required to improve antibacterial use.

The predominance of CRKP and CRAB isolates in current study, could be due to the invasive device-associated infections during ICU stay due to mechanical ventilation and empirical antibiotic use. Also indicated was an environmental source, pointing to poor hand hygiene and poor infection control practices.

During COVID-19 pandemic, due to fear of COVID-19 approximately all healthcare workers use gloves as part of personal protective equipment and do not feel the need to perform hand hygiene before and after patient care, and there is a lack of concern for patient to patient transmission of XDR pathogens in hospitalized patients (Vijay et al., 2021). As most of 
the bacterial infections in the present study were XDR pathogens nosocomial in origin, it highlighted poor infection control measures (e.g., hand washing, glove changing, and meticulous cleaning of equipment), that could have led to crosstransmission of XDR pathogens during patient care.

Our study has several limitations. First, it is a single-center retrospective study from an academic hospital in the epicenter of the COVID-19 pandemic, and the data collected were limited. Therefore, our results may not be generalizable to other centers with a different bacterial ecology. Second, during the study period, molecular diagnostic tests (e.g., PCR) were not available for detection of atypical pathogens, such as Mycoplasma pneumoniae. Therefore, we might have missed atypical secondary bacterial infection identified and other respiratory pathogen infections usually diagnosed by PCR. Third, due to the lack of a mycology laboratory in our hospital, it was not possible to accurately identify fungal infections. So, the true incidence of fungal infections remains unclear. Finally, a differential diagnosis of GGO pattern was not performed.

In conclusion, the superinfection among patients admitted to the ICU wards is a serious problem in the COVID-19 pandemic, which can lead to increasing the disease severity and mortality. Herein, we report on a $11.9 \%$ rate of secondary bacterial infection at ICU admission of adult patients with severe COVID-19, mostly with CRKP and CRAB that associated with high mortality rates $(83 \%)$. These data are also in line with the previously documented high mortality associated with these pathogens (Balkhair et al., 2019). With increasing cases of COVID-19 in Iran, there is a need to reinforce the principles of antimicrobial stewardship and infection control.

\section{REFERENCES}

Arayasukawat, P., So-Ngern, A., Reechaipichitkul, W., Chumpangern, W., Arunsurat, I., Ratanawatkul, P., et al. (2012). Microorganisms and Clinical Outcomes of Early- and Late-Onset Ventilator-Associated Pneumonia at Srinagarind Hospital, a Tertiary Center in Northeastern Thailand. BMC Pulm Med. 21, 1-8. doi: 10.1186/s12890-021-01415-8

Balkhair, A., Al-Muharrmi, Z., Al'adawi, B., Al Busaidi, I., Taher, H. B., AL-Siyabi, T., et al. (2019). Prevalence and 30-Day All-Cause Mortality of Carbapenem-and Colistin-Resistant Bacteraemia Caused by Acinetobacter Baumannii, Pseudomonas Aeruginosa, and Klebsiella Pneumoniae: Description of a Decade-Long Trend. Int. J. Infect. Dis. 85, 10-15. doi: 10.1016/j.ijid.2019.05.004

Baskaran, V., Lawrence, H., Elansbury, L., Webb, K., Safavi, S., Zainuddin, N., et al. (2021). Co-Infection in Critically Ill Patients With COVID-19: An Observational Cohort Study From England. J. Med. Microb. 70, 1-9. doi: 10.1099/jmm.0.001350

Bolourchi, N., Shahcheraghi, F., Giske, C. G., Nematzadeh, S., Noori Goodarzi, N., Solgi, H., et al. (2021). Comparative Genome Analysis of Colistin-Resistant OXA-48-Producing Klebsiella Pneumoniae Clinical Strains Isolated From Two Iranian Hospitals. Ann. Clin. Microbiol. Antimicrob. 74, 1-11. doi: 10.1186/ s12941-021-00479-y

Clinical and Laboratory Standards Institute [CLSI] (2017). Performance Standards for Antimicrobial Susceptibility Testing. 27th Edn (Wayne, PA: Clinical and Laboratory Standards Institute).

Contou, D., Claudinon, A., Pajot, O., Micaëlo, M., Flandre, P. L., Dobert, M., et al. (2020). Bacterial and Viral Co-Infections in Patients With Severe SARS-CoV-2 Pneumonia Admitted to a French ICU. Ann. Intensive Care 10, 1-9. doi: $10.1186 /$ s13613-020-00736-x

\section{DATA AVAILABILITY STATEMENT}

The original contributions presented in the study are included in the article/supplementary material. Further inquiries can be directed to the corresponding author.

\section{ETHICS STATEMENT}

The studies involving human participants were reviewed and approved by No. IR.MUI.MED.REC.1400.453. Written informed consent for participation was not required for this study in accordance with the national legislation and the institutional requirements.

\section{AUTHOR CONTRIBUTIONS}

Project design: HS, SP and EK. Investigation: HS, MS and MA. Data analysis: HS. Writing-Original draft: HS, HT, HM, FS, and BA. Writing-Review \& editing: HS, SP, EK, HT, HM, RS, $\mathrm{BA}$, and FK. All authors listed have made a substantial, direct, and intellectual contribution to the work and approved it for publication.

\section{FUNDING}

This work was funded by research grants from the Isfahan University of Medical Sciences (project no. 50485).

Corman, V. M., Landt, O., Kaiser, M., Molenkamp, R., Meijer, A., Chu, D. K. W. et al. (2020). Detection of 2019 Novel Coronaviru-Ncov) by Real-Time RTPCR. Euro Surveill 25, 2000045. doi: 10.2807/1560-7917.ES.2020.25.3.2000045

EUCAST. (2017). Breakpoint Tables for Interpretation of MICs and Zone Diameters. Version 7.0. Available at: http://www.eucast.org/clinical_ breakpoints/ (Accessed February 24, 2017).

Jakribettu, R., Boloor, R., and Suresh, S. (2016). Comparison of Microbiological Profile of Pathogens Isolated From Early-Onset and Late-Onset VentilatorAssociated Pneumonia in a Tertiary Care Center. Trop. J. Med. Res. 19, 14-19. doi: 10.4103/1119-0388.172073

Langford, B., So, M., Raybardhan, S., Leung, V., Westwood, D., MacFadden, D. R., et al. (2020). Bacterial Co-Infection and Secondary Infection in Patients With COVID-19: A Living Rapid Review and Meta-Analysis. Clin. Microbiol. Infect. 26, 1622-1629. doi: 10.1016/j.cmi.2020.07.016

Lansbury, L., Lim, B., Baskaran, V., and Lim, W. S. (2020). Co-Infections in People With COVID-19: A Systematic Review and Meta-Analysis. J. Infect 81, 266275. doi: 10.1016/j.jinf.2020.05.046

Lehmann, C. J., Pho, M. T., Pitrak, D., Ridgway, J., and Pettit, N. N.. (2020). Community Acquired Co-Infection in COVID-19: A Retrospective Observational Experience. Clin. Infect. Dis. 72, 1450-1452. doi: 10.1093/cid/ ciaa902

Nazari, M., Azizi, O., Solgi, H., Fereshteh, S., Shokouhi, S., and Badmasti, F. (2021). Emergence of Carbapenem Resistant Acinetobacter Baumannii Clonal Complexes CC2 and CC10 Among Fecal Carriages in an Educational Hospital. Int. J. Environ. Health Res. 15, 1-12. doi: 10.1080/09603123.2021.1892036

Neto, A. G. M., Bryan, L. O. K., Wattoo, A., Salacup, G., Pelayo, J., Dejoy, R., et al. (2020). Bacterial Infections and Patterns of Antibiotic Use in Patients With COVID - 19. J. Med. Virol. 18, 1-7. doi: 10.1002/jmv.26441 
Nori, P., Cowman, K., Chen, V., Bartash, R., Szymczak, W., Madaline, T., et al. (2020). Bacterial and Fungal Coinfections in COVID-19 Patients Hospitalized During the New York City Pandemic Surge. Infect. Control Hosp. Epidemiol. 42, 84-88. doi: 10.1017/ice.2020.368

Patel, A., Emerick, M., Cabunoc, M. K., Williams, M. H., Preas, M. A., Schrank, G., et al. (2021). Rapid Spread and Control of Multidrug-Resistant Gram-Negative Bacteria in COVID-19 Patient Care Units. Emerg. Infect. Dis. 27, 1234-1236. doi: 10.3201/eid2704.204036

Russell, C. D., Fairfield, C. J., Drake, T. M., Turtle, L., Seaton, A., Wootton, D. G., et al. (2021). Co-Infections, Secondary Infections, and Antimicrobial Use in Patients Hospitalised With COVID-19 During the First Pandemic Wave From the ISARIC WHO CCP-UK Study: A Multicentre, Prospective Cohort Study. Lancet Microbe. 2, e354-65. doi: 10.1016/S2666-5247(21)00090-2

Sharifipour, E., Shams, S., Esmkhani, M., Khodadadi, J., Fotouhi-Ardakani, R., Koohpaei, A., et al. (2020). Evaluation of Bacterial Co-Infections of the Respiratory Tract in COVID-19 Patients Admitted to ICU. BMC Infect. Dis. 20, 1-7. doi: 10.1186/s12879-020-05374-Z

Solgi, H., Ghafarzadeh, H., and Shahcheraghi, F. (2017). Evaluation of Phenotypic and Genotypic Carbapenemase Genes in Gram-Negative Bacteria Resistant to Carbapenem and Determining Their Antibiotic Resistance. J. Isfahan Med. Sch. 34, 1290-1296.

Solgi, H., Shahcheraghi, F., Bolourchi, N., and Ahmadi, A.. (2020). Molecular Characterization of Carbapenem-Resistant Serotype K1 Hypervirulent Klebsiella Pneumoniae ST11 Harbouring bla $\mathrm{NDM}^{-1}$ and $b l a_{\mathrm{OXA}^{-} 48}$ Carbapenemases in Iran. Microb. Pathog. 149, 104507. doi: 10.1016/j.micpath.2020.104507

Turton, J. F., Woodford, N., Glover, J., Yarde, S., Kaufmann, M. E., Pitt, T., et al. (2006). Identification of Acinetobacter Baumannii by Detection of the Bla OXA-51 -Like Carbapenemase Gene Intrinsic to This Species. J. Clin. Microbiol. 44, 2974-2976. doi: 10.1128/JCM.01021-06

Vijay, S., Bansal, N., Kumar Rao, B., Veeraraghavan, B., Rodrigues, C., Wattal, C., et al. (2021). Secondary Infections in Hospitalized COVID-19 Patients: Indian Experience. Infect. Drug Resist. 14, 1893-1903. doi: 10.2147/IDR.S299774

Wang, Z., Yang, B., Li, Q., Wen, L., and Zhang, R.. (2020). Clinical Features of 69 Cases With Coronavirus Disease 2019 in Wuhan, China. Clin. Infect. Dis. 71, 769-777. doi: 10.1093/cid/ciaa272
WHO. Covid-19 Dashboard 2020. Available at: https://covid19.who.int/?gclid= CjwKCAjw8df2BRA3EiwAvfZWaJWnmCWZBUjJdJZGVdH4hGEN u8orjqQTHDsIst5u_gYXoQcl8sS_ZxoCIxEQAvD_BwE.

World Health Organization. (2020). Clinical Management of Severe Acute Respiratory Infection (SARI) When COVID-19 Disease is Suspected: Interim Guidance. Available at: https://www.who.int/publications-detail/clinicalmanagement-of-severe-acuterespiratory-infection-when-novel-coronavirus(ncov)-infection-is-suspected (Accessed Mar 29, 2020).

Wu, J., Liu, J., Zhao, X., Liu, C., Wang, W., Wang, D., et al. (2020). Clinical Characteristics of Imported Cases of Coronavirus Disease 2019 (COVID-19) in Jiangsu Province: A Multicenter Descriptive Study. Clin. Infect. Dis. 71, 706712. doi: $10.1093 / \mathrm{cid} / \mathrm{ciaa} 199$

Yang, X., Yu, Y., Xu, J., Shu, H., Xia, J., Liu, H., et al. (2020). Clinical Course and Outcomes of Critically Ill Patients With SARS-CoV-2 Pneumonia in Wuhan, China: A Single-Centered, Retrospective, Observational Study. Lancet Respir. Med. 8, 475-481. doi: 10.1016/S2213-2600(20)30079-5

Conflict of Interest: The authors declare that the research was conducted in the absence of any commercial or financial relationships that could be construed as a potential conflict of interest.

Publisher's Note: All claims expressed in this article are solely those of the authors and do not necessarily represent those of their affiliated organizations, or those of the publisher, the editors and the reviewers. Any product that may be evaluated in this article, or claim that may be made by its manufacturer, is not guaranteed or endorsed by the publisher.

Copyright (C) 2022 Pourajam, Kalantari, Talebzadeh, Mellali, Sami, Soltaninejad, Amra, Sajadi, Alenaseri, Kalantari and Solgi. This is an open-access article distributed under the terms of the Creative Commons Attribution License (CC BY). The use, distribution or reproduction in other forums is permitted, provided the original author(s) and the copyright owner(s) are credited and that the original publication in this journal is cited, in accordance with accepted academic practice. No use, distribution or reproduction is permitted which does not comply with these terms. 